\title{
Distinct actions of intermittent and sustained $\beta$-adrenoceptor stimulation on cardiac remodeling
}

\author{
MA XiaoWei ${ }^{1,3}$, SONG Yao ${ }^{1,3}$, CHEN Chao ${ }^{1,3}$, FU YongNan ${ }^{1,3}$, SHEN Qiang $^{2,3}$, \\ LI ZiJian ${ }^{1,3}$ \& ZHANG YouYi ${ }^{1,3^{*}}$ \\ ${ }^{1}$ Institute of Vascular Medicine, Peking University Third Hospital, Beijing 100191, China; \\ ${ }^{2}$ Institute of Cardiovascular Science, Peking University, Beijing 100191, China; \\ ${ }^{3}$ Key Laboratory of Molecular Cardiovascular Sciences, Ministry of Education, Beijing 100191, China
}

Received April 14, 2011; accepted May 10, 2011

\begin{abstract}
Heart disease is associated with increased sympathetic nerve activity and elevated levels of circulating catecholamines, resulting in chronic stimulation of the $\beta$-adrenergic receptors ( $\beta$-AR) and consequent pathological cardiac remodeling. Experimentally, chronic administration of the $\beta$-AR agonist isoproterenol (ISO) has been most commonly used to model $\beta$-AR-induced cardiac remodeling. However, it remains unclear whether $\beta$-AR-mediated cardiac remodeling and dysfunction differs between sustained versus pulsatile (intermittent) exposure to a $\beta$-agonist. Here, we compare the effects of intermittent versus sustained administration of ISO on cardiac remodeling and function in mice. Animals were administered $5 \mathrm{mg}(\mathrm{kg} \mathrm{d})^{-1}$ ISO for 2 weeks either by daily subcutaneous injection, or continuous infusion via an implanted osmotic minipump. Cardiac function and remodeling were determined by echocardiography, micromanometry and histology. Moreover, Western blotting and quantitative real-time polymerase chain reaction (qRT-PCR) were utilized to define the proteins and genes involved. Both sustained and intermittent administration of ISO resulted in a similar degree of cardiac hypertrophy (16\% and 19\%, respectively). However, mice receiving ISO by daily injection developed more severe ventricular systolic and diastolic dysfunction and myocardial fibrosis compared with mice receiving ISO via the osmotic minipump. The disparity in results between the delivery methods is suggested to be due, at least in part, to increased expression of fibrogenic factors, including connective tissue growth factor (CTGF) and NADPH oxidase (NOX4), in mice receiving intermittent application of ISO. In summary, compared with sustained exposure to a $\beta$-AR agonist, intermittent $\beta$-AR stimulation leads to more severe cardiac dysfunction and fibrosis. These findings not only further our understanding of $\beta$-AR function in the setting of cardiac pathophysiology, but also highlight that significant differences can result dependent upon the mode of experimental $\beta$-AR stimulation in inducing cardiomyopathy.
\end{abstract}

$\beta$-adrenoceptor, reactive oxygen species, NADPH oxidase, cardiac hypertrophy, cardiac fibrosis

Citation: $\quad$ Ma X W, Song Y, Chen C, et al. Distinct actions of intermittent and sustained $\beta$-adrenoceptor stimulation on cardiac remodeling. Sci China Life Sci, 2011, 54: 493-501, doi: 10.1007/s11427-011-4183-9

Symptomatic heart failure and sudden cardiac death are major causes of morbidity and mortality worldwide. Increased cardiac sympathetic nerve activity is a predominant characteristic of patients suffering heart failure [1,2], and prolonged stimulation of the $\beta$-adrenergic neurohormonal

*Corresponding author (email: zhangyy@bjmu.edu.cn) axis has been shown to contribute to the progression of heart failure and mortality in both animal models and human patients $[3,4]$. Excessive $\beta$-adrenergic receptor $(\beta$-AR) stimulation has been reported to induce cardiac myocyte apoptosis both in vivo and in vitro, as well as induce cardiac chamber remodeling associated with hypertrophy and/or fibrosis [5-7]. In the clinical setting, both sustained and 
intermittent patterns of $\beta$-AR stimulation have been described.

Hypertension is a prevalent cardiovascular disorder with a complex etiology. Currently, the pathophysiological mechanisms of essential hypertension are poorly understood; however, the disease is often associated with increased sympathetic nerve activity, elevated levels of circulating catecholamines and sustained $\beta$-AR stimulation $[8,9]$. Transient $\beta$-AR stimulation following stress has also been reported to cause acute cardiomyopathy. Stress-induced cardiomyopathy is characterized by acute, but rapidly reversible, left ventricular systolic dysfunction in the absence of atherosclerotic coronary artery disease. Current reports suggest that stress-induced cardiomyopathy is triggered by intense psychological or physical stressors [10]. Moreover, as catecholamine levels are elevated during times of stress, it is proposed that the resultant cardiomyopathy is predominantly due to transient $\beta$-AR stimulation $[11,12]$.

The use of isoproterenol (ISO), a powerful nonselective $\beta$-AR agonist, to model myocardial injury in the experimental setting was initiated by Rona et al. [13] and validated in subsequent studies [14,15]. ISO treatment causes significant cardiac remodeling in rats, associated with severe myocardial hypertrophy and injury $[16,17]$, as well as apoptosis of cardiomyocytes [6,17]. Notably, ISO can be administered by different means, either by repeated subcutaneous injection (intermittent $\beta$-AR stimulation) or continuous subcutaneous infusion (sustained $\beta$-AR stimulation). Currently it remains unclear whether the extent of cardiac remodeling and dysfunction following $\beta$-AR stimulation differs between intermittent exposure to a $\beta$-agonist and sustained $\beta$-agonist administration.

The mitogen-activated protein (MAP) kinase superfamily, including extracellular signal-regulated kinase (ERK), c-Jun NH2-terminal kinase (JNK) and p38-kinase, is predominantly stimulated by growth factors, cellular stress or inflammatory cytokines. Of note, there is increasing evidence that MAP kinases play specific roles during the early development of cardiac hypertrophy [18-20]. Activation of the mammalian target of rapamycin (mTOR)/protein S6 kinase (p70S6k) signaling pathway by phenylephrine (PE) and angiotensin-II was found to stimulate protein synthesis and promote cardiac hypertrophy [21,22]. Furthermore, application of ISO has been demonstrated to increase the phosphorylation of mTOR and p70S6k in vitro [23,24], implying the potential for ISO-induced cardiac hypertrophy to be mediated by the mTOR/p70S6k signaling pathway.

Cardiac hypertrophy and myocardial fibrosis are also associated with elevated generation of reactive oxygen species (ROS) $[25,26]$ and connective tissue growth factor (CTGF) is known to play an important role in the myocardial fibrosis process. There is evidence that ROS-related signaling pathways are involved in CTGF synthesis process which is important in AngII-induced cardiac fibrosis [27]. However, the role of CTGF in ISO-induced cardiac remodeling is not clearly understood.

The present study sought to determine whether different modes of $\beta$-AR stimulation impart differential actions on cardiac remodeling. The study further explored the potential mechanisms underlying these effects.

\section{Materials and methods}

\subsection{Animal model}

All study protocols conformed to the Animal Management Rules of China (Documentation No. 55, 2001, Ministry of Health, China). All experiments were approved by the Committee for Ethics of Animal Experiments and were conducted in accordance with the Guidelines for Animal Experiments, Peking University Health Science Center. Ten-week-old male BALB/c mice were obtained from the Animal Department of the Peking University Health Science Center. Mice received isoproterenol (5 mg $\left.(\mathrm{kg} \mathrm{d})^{-1}\right)$, dissolved in $0.1 \mathrm{mmol} \mathrm{L}^{-1}$ ascorbic acid; Sigma-Aldrich, St. Louis, USA) or vehicle (ascorbic acid, i.e. vitamin C, VC, $0.1 \mathrm{mmol} \mathrm{L}^{-1}$ ) via either daily subcutaneous injection or continuous infusion by implanting an osmotic minipump (Alzet model 1002, Durect Corp., Cupertino, USA) subcutaneously in the neck. Insertion of the minipump was performed under anesthesia using tribromoethanol $\left(150 \mathrm{mg} \mathrm{kg}^{-1}\right.$, i.p.; Sigma-Aldrich, St. Louis, USA). Mice were randomly divided into the four treatment groups: (i) VC s.c. group: mice received daily subcutaneous injection of vehicle $(n=8)$; (ii) ISO s.c. group: mice received daily subcutaneous injection of ISO ( $n=8)$; (iii) VC pump group: mice received continuous infusion of vehicle using an osmotic pump $(n=8)$; (iv) ISO pump group: mice received continuous infusion of ISO using an osmotic pump $(n=16)$. Echocardiography was used to evaluate heart rate, cardiac structure and function at specific time points following ISO administration. On day 14, echocardiography and hemodynamic analyses were performed for the final time before mice were sacrificed. As a side, osmotic pumps were removed from eight of the ISO-infused mice, and cardiac function was evaluated three days after pump removal.

\subsection{Echocardiography and evaluation of left ventricu- lar hemodynamics}

Mice were anaesthetized using $1.5 \%$ isoflurane (Baxter Healthcare Corporation, New Providence, USA). Echocardiographic images were obtained using a Visualsonics highresolution Vevo 770 system (VisualSonics, Incorporated, Toronto, Canada). Two-dimensional parasternal long-axis views and short-axis views were obtained at the level of the papillary muscle. Diastolic left ventricular posterior wall thickness (LVPWd) and systolic left ventricular posterior 
wall thickness (LVPWs) were measured. Ejection fraction (EF) and fractional shortening (FS) were calculated from these parameters. All measurements were averaged from three consecutive cardiac cycles. To measure aortic and left ventricular (LV) pressure, a 1.4-F micromanometer conductance catheter (SPR-835; Millar Instruments, Incorporated, Houston, USA) was introduced through the right common carotid artery into the ascending aorta and then advanced into the left ventricle as previously described [28].

\subsection{Histology}

Following sacrifice hearts were harvested and perfused in retrograde with cold phosphate-buffered saline (PBS), fixed with $4 \%$ paraformaldehyde overnight, and embedded in paraffin. Serial sections ( $6 \mu \mathrm{m}$ thick) were stained with hematoxylin and eosin (HE) for morphological analysis, or Sirius red for the detection of fibrosis. For morphometrical analysis, photographs of LV sections cut from the same location of each heart were observed under $\times 400$ magnification. Cross-sectional images of cardiac myocytes $(n=100$ cells from each heart) were digitized using the Leica Q550 IW imaging workstation (Leica Microsystems Imaging Solutions Ltd., Cambridge, UK). Interstitial collagen type I and III were visualized using Sirius red stain under bright field and polarized light microscopy, and the cardiac collagen volume fraction was calculated as the ratio of the stained fibrotic area to total myocardial area.

\subsection{Quantitative real-time PCR}

Total RNA was isolated from heart tissue using Trizol Reagent (Invitrogen). Relative quantitation by real-time PCR was performed using SYBR Green to detect PCR products in real time with ABI PRISM 7700 Sequence Detection System (Applied Biosystems). The sequences of oligonucleotide primers were as follows: ANF, 5'-GCCCTGAGTGAGCAGACTG-3' (forward) and 5'-CGGAAGCTGTTGCAGCCTA-3' (reverse); Collagen-I, 5'-GTAACTTCGTGCCTAGCAACA-3' (forward) and 5'-CCTTTGTCAGAATACTGAGCAGC-3' (reverse); Collagen-III, 5'-CCTGGCTCAAATGGCTCAC-3' (forward) and 5'-CAGGACTGCCGTTATTCCCG-3' (reverse); CTGF, 5'-GGGCCTCTTCTGCGATTTC-3' (forward) and 5'-ATCCAGGCAAGTGCATTGGTA-3' (reverse); NOX4， 5'-ACTTTTCATTGGGCGTCCTC-3' (forward) and 5'-AGAACTGGGTCCACAGCAGA-3' (reverse); GAPDH, 5'-TCCTGGTATGACAATGAATACGGC-3' (forward) and 5'-TCTTGCTCAGTGTCCTTGCTGG-3' (reverse). GAPDH RNA was amplified as an internal control. The cell cycle was: $94^{\circ} \mathrm{C}$ for 2 min, followed by 40 cycles at $94^{\circ} \mathrm{C}$ for $15 \mathrm{~s}, 58^{\circ} \mathrm{C}$ for $15 \mathrm{~s}$ and $72^{\circ} \mathrm{C}$ for $30 \mathrm{~s}$.

\subsection{Western blot analysis}

Protein expression was examined by Western blot analysis as previously described [29]. Proteins were electrophoresed and analyzed using anti-phospho-ERK1/2, anti-phosphoJNK1/2, anti-phospho-p38, anti-phospho-mTOR, anti-phospho-p70S6k, anti-JNK1/2, anti-mTOR, anti-p70S6k (Cell Signaling Technology Incorporated, Danvers, MA, USA), anti-ERK1/2, anti-p38, anti-CTGF, anti-eIF5 (Santa Cruz, CA, USA), and anti-NOX4 (Abcam Inc., Cambridge, MA).

\subsection{Measurement of ROS}

The amount of ROS expression in heart tissue was visualized by dihydroethidium (DHE) (Invitrogen Molecular Probes, Eugene, OR, USA) which gives red fluorescence when oxidized to ethidium in the presence of ROS. Serial sections $(6 \mu \mathrm{m})$ of paraformaldehyde-fixed heart tissue were deparaffinized, rehydrated and incubated with $5 \mu \mathrm{mol} \mathrm{L} \mathrm{L}^{-1}$ DHE for $30 \mathrm{~min}$ at $37^{\circ} \mathrm{C}$ under $5 \% \mathrm{CO}_{2}$ in air. Sections were analyzed using a Leica digital camera mounted on a fluorescent microscope attached to a personal computer running Leica software.

\subsection{Statistical analysis}

All data are presented as the mean \pm SE. Inter-group comparisons were performed by a Student's paired two-tailed $t$-test or two-way ANOVA (Prism 4, GraphPad Software Incorporate, La Jolla, CA, USA). The Bonferroni test was used for post hoc analysis where significant variance occurred in the ANOVA. $P<0.05$ was considered statistically significant.

\section{Results}

\subsection{Effects of ISO on heart rate}

ISO caused a significant increase in the heart rate (HR) of mice. After peaking at $1 \mathrm{~min}$ ( $45 \%$ increase), the heart rate of mice receiving intermittent application of ISO decreased gradually to basal levels after $30 \mathrm{~min}$. In contrast, animals receiving ISO by the minipump had a rapid although less pronounced increase in HR at $1 \mathrm{~min}(P<0.01$ versus ISO injection), which was sustained and increased slightly over the experimental period, peaking at $26 \%$ above control (Figure 1).

\subsection{ISO induces cardiac hypertrophy}

Heart sections stained with HE revealed that administering ISO by injection or the pump significantly increased the mean cardiomyocyte cross-sectional area by $68 \%$ and $63 \%$ respectively, compared with their respective controls. No significant difference was found between ISO groups (Figures $2 \mathrm{~A}$ and $\mathrm{B}$ ). Mice receiving ISO by injection or the pump both exhibited a significant increase in the ratio of heart weight to body weight (HW/BW) compared with ve- 


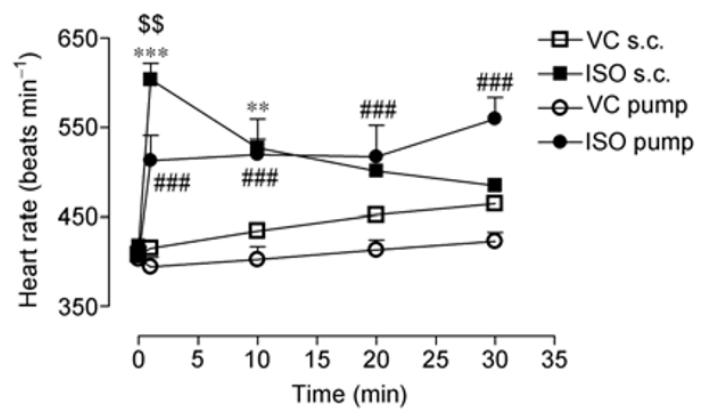

Figure 1 The effect of ISO on heart rate is dependent upon its mode of application. The heart rate of mice at baseline, 1, 10, 20, and $30 \mathrm{~min}$ after injections (s.c.) or continuous infusion (pump) of ISO or vehicle (VC). ** $P<0.01$, *** $P<0.001$ vs. VC s.c.; \#\#\# $P<0.001$ vs. VC pump; $\$ \$ P<0.01$ vs. ISO pump; $n=5$ per group.

hicle (16\% and $19 \%$ respectively); however the two ISO groups did not significantly differ (Figure 2C). ISO delivery also increased ANF mRNA expression with no significant difference between the two delivery methods (Figure 2D).

\subsection{ISO-induced cardiac fibrosis}

Observing the morphology, collagen deposition increased in response to ISO treatment by both modes of delivery. Mice receiving intermittent ISO injections exhibited significantly greater collagen deposition compared with the ISO pump group (Figures 3A and B). Quantitative analysis revealed cardiac interstitial fibrosis was markedly increased by $93 \%$ and 53\% in ISO-injected and ISO-pump animals compared with their controls, respectively. Significantly, fibrosis was $54 \%$ greater in ISO-injected mice compared with ISO-pump mice $(P<0.05$, Figure $3 \mathrm{C})$. Consistent with this, quantitative RT-PCR analysis demonstrated that the relative mRNA content of myocardial collagen-I and collagen-III was significantly increased in ISO-treated mice, with ISO-injected mice displaying markedly higher levels of collagen-I and -III mRNA compared with the ISO pump group (Figures 3D and $\mathrm{E})$.

\subsection{Effect of ISO on cardiac function}

Echocardiography revealed that FS and EF were significantly increased after 2 days of ISO treatment under both intermittent and continuous infusion protocols. Mice subjected to continuous infusion of ISO maintained FS and EF above control levels across the entire experimental period. In contrast, mice receiving injections of ISO displayed a decline in FS and EF following day 2, becoming consistent with basal levels on day 4 , before further reducing to become significantly below control levels at the end of the experimental period (Figures 4A, B and C). Hemodynamic data were consistent with the echocardiograph findings where mice receiving ISO by injection exhibited a significant reduction in left ventricular force of contraction $(+\mathrm{dP} / \mathrm{dt})$ and relaxation $(-\mathrm{dP} / \mathrm{dt})$ (Figures $4 \mathrm{D}$ and $\mathrm{E})$, to-
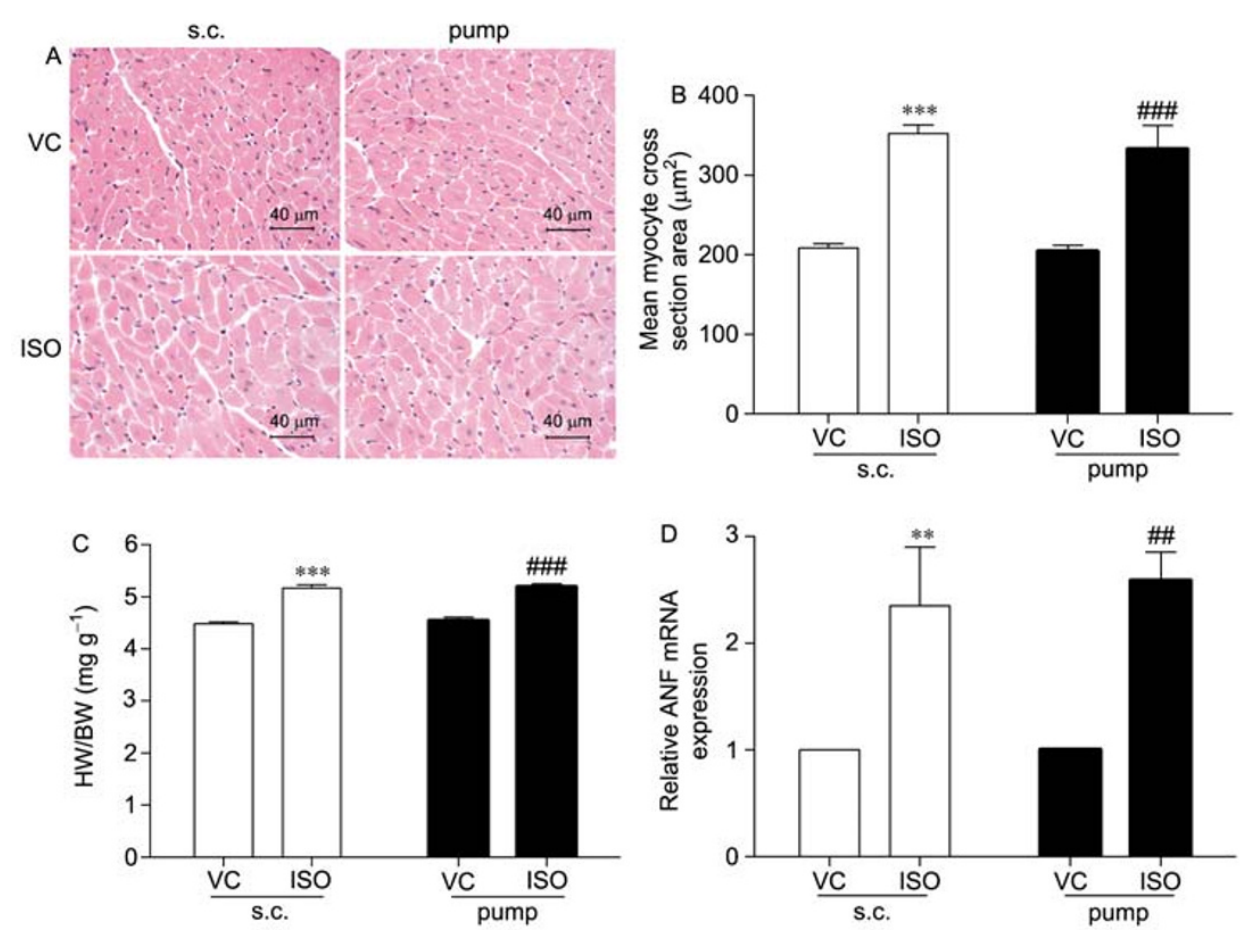

Figure 2 ISO-induced cardiac hypertrophy in vivo. A, Representative micrographs of HE-stained sections of the left ventricle (LV). B, Mean myocyte cross-sectional area calculated from HE-stained sections of LVs $(n=8)$. C, The ratio of heart weight $(\mathrm{HW})$ to body weight $(\mathrm{BW})(n=8)$. D, Expression of atrial natriuretic factor (ANF) mRNA normalized to that of GAPDH $(n=6)$. s.c., subcutaneous injection; pump, continuous infusion via the osmotic pump. $* * P<0.01$, *** $P<0.001$ vs. VC s.c.; \#\# $P<0.01$, \#\#\# $P<0.001$ vs. the VC pump. 

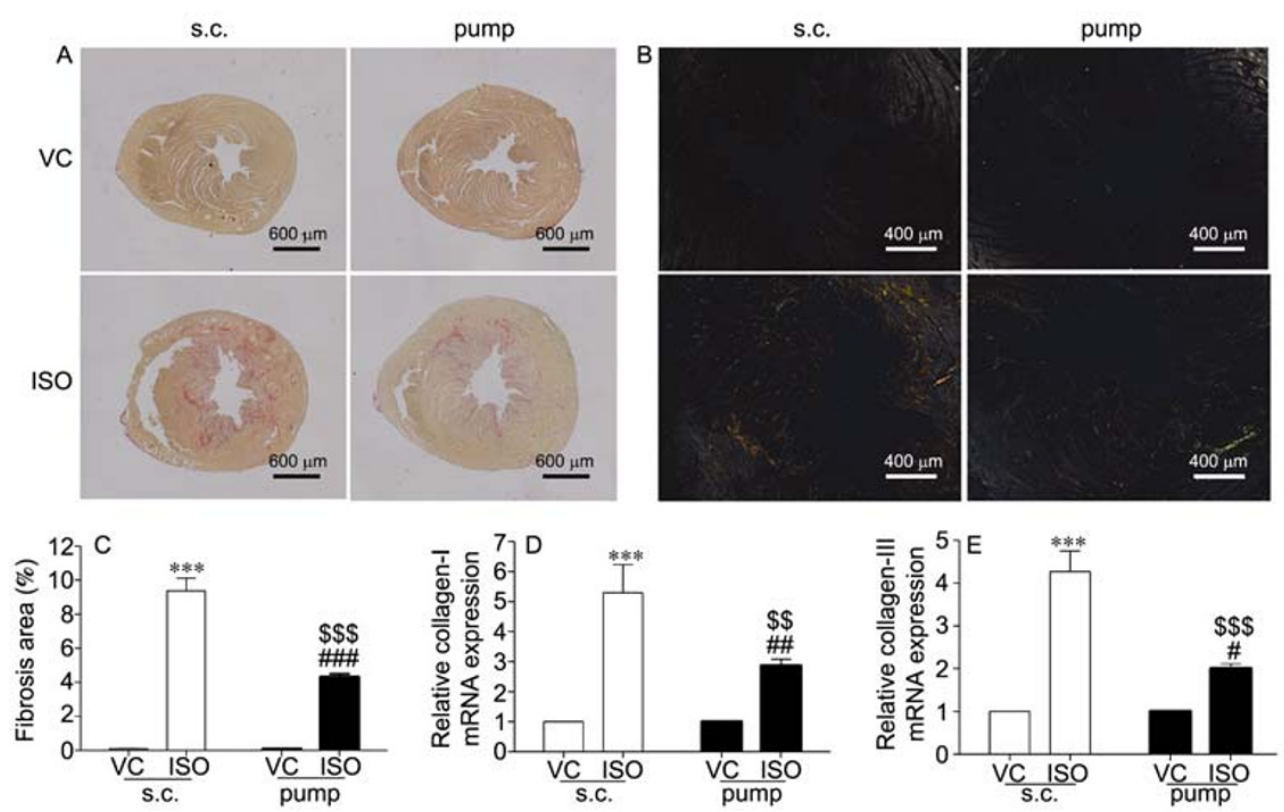

Figure 3 ISO-induced cardiac fibrosis in vivo. A, Administration of ISO significantly increased the amount of collagen (red staining) within the cardiac interstitium, visualized under bright field microscopy (A) and polarized light microscopy (B) (yellow and green stain represent collagen). Quantitation of these stains provided the average interstitial collagen content $(\mathrm{C}, n=8)$. qRT-PCR performed on LV tissue confirmed an increase in collagen-I and -III mRNA (D and E respectively), both normalized to GAPDH (both $n=6$ ). s.c., subcutaneous injection; pump, continuous infusion via the osmotic pump. *** $P<0.001$ vs. VC s.c.; $\# P<0.05$, \#\# $P<0.01$, \#\#\# $P<0.001$ vs. VC pump; $\$ P<0.01, \$ \$ \$<0.001$ ISO pump vs. ISO s.c.
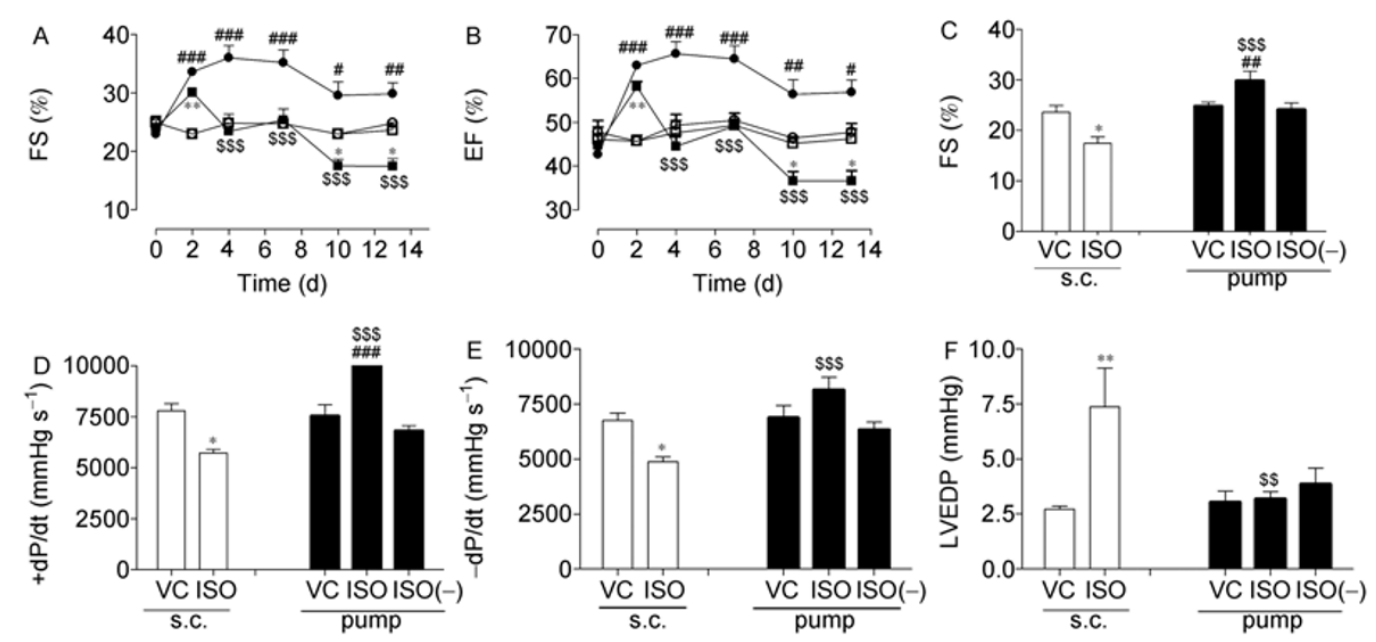

Figure 4 Effect of ISO on cardiac function. Fractional shortening (FS) (A) and ejection fraction (EF) (B) were determined by echocardiography at specific time points following ISO treatment ( $\square$ VC s.c., OVC pump, $\square$ ISO s.c., $\bullet$ ISO pump; $n=8$ ), and FS again at the end of the study period (C). D and E indicate the effect of ISO treatment on LV force of contraction $(+\mathrm{dP} / \mathrm{dt})$ and relaxation $(-\mathrm{dP} / \mathrm{dt})$ at the end of the study $(n=8)$. Left ventricular end-diastolic pressure (LVEDP) was also obtained by hemodynamic analysis at the end of the study period (F, $n=8)$. ISO(-), ISO removal group; s.c., subcutaneous injection; pump, continuous infusion via the osmotic pump. ${ }^{*} P<0.05$, ** $P<0.01$ vs. VC s.c.; \# $P<0.05$, \# $P<0.01$, \#\#\# $P<0.001$ vs. the VC pump; $\$ P<0.01$, $\$ \$ P<0.001$ ISO pump vs. ISO s.c.

gether with increased left ventricular end-diastolic pressure (LVEDP) (Figure 4F) compared with both ISO-pump and control vehicle mice. No significant differences in systolic function were found when comparing mice that received a continuous infusion of ISO having the pump removed for 3 days and vehicle-treated mice (Figures 4C, D, E and F).

\subsection{Effects of ISO treatment on mTOR/p70S6k, MAPK and CTGF expression}

Cardiac tissue from ISO-treated mice (both injected and continuously infused) exhibited a 2 -fold increase in phospho-mTOR, and a 5-fold increase in the phosphorylation of its downstream molecule, p70S6k (Figure 5A). The level of 

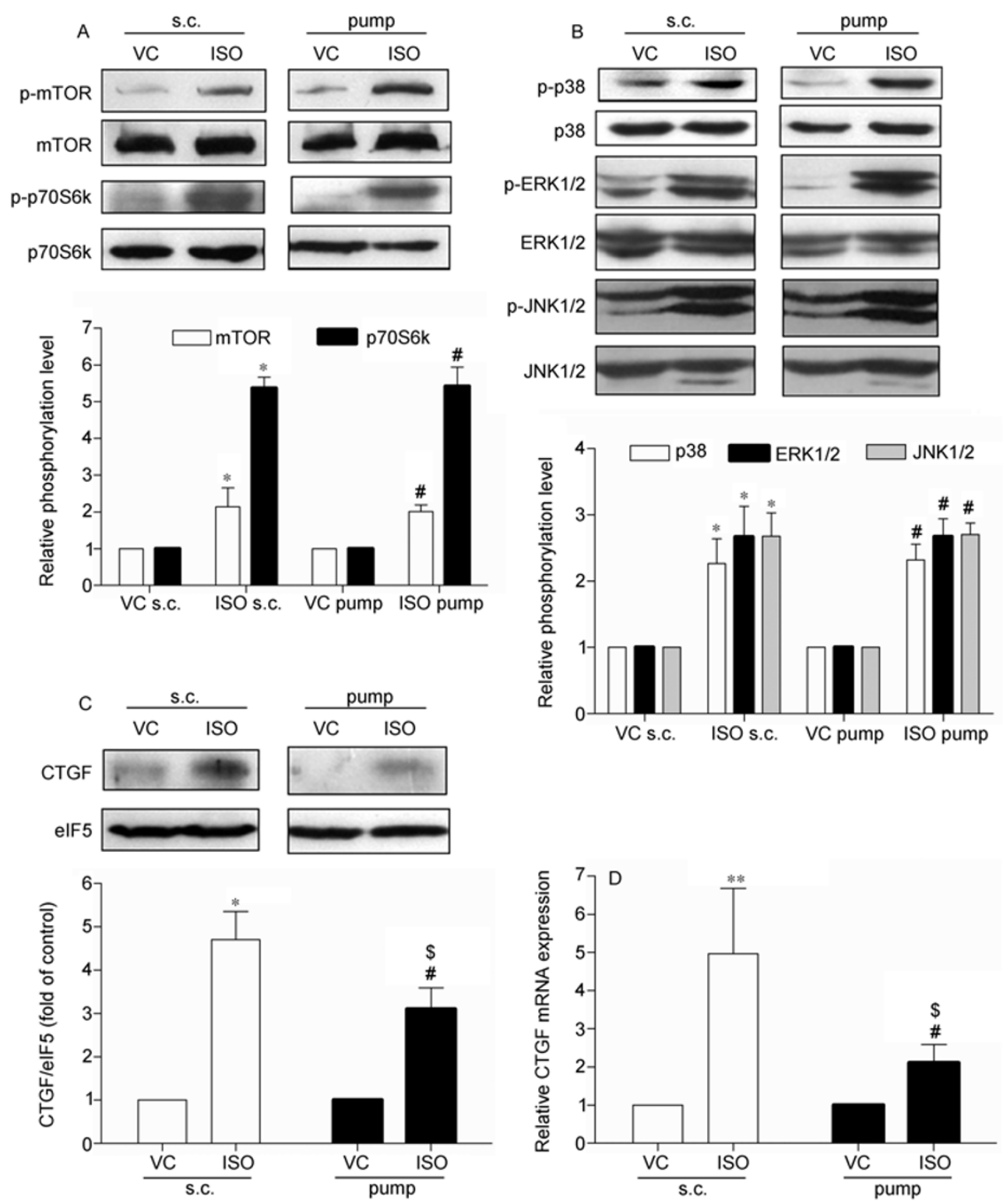

Figure 5 Effect of ISO on mTOR, p70S6k, MAPK and CTGF expression. Western blotting provided the expression levels of phosphorylated and total protein expression of mTOR and p70S6k $(n=4)(\mathrm{A})$, MAPK $(n=4)(\mathrm{B})$, and CTGF $(n=4)(\mathrm{C})$. mRNA expression of CTGF was also determined by RT-PCR $(n=6)(\mathrm{D})$. For Figures A, B and C, the upper panels provide representative blots of the quantified and pooled findings (lower graphs). s.c., subcutaneous

injection; pump, continuous infusion via the osmotic pump. * $P<0.05$, ** $P<0.01$ vs. VC s.c.; $\# P<0.05$ vs. VC pump; $\$ P<0.05$ ISO pump vs. ISO s.c.

phosphorylation of ERK1/2, JNK1/2, and p38 all increased to a similar extent under both ISO-delivery methods after 14 days of administration (Figure 5B). CTGF mRNA and protein expression were also significantly elevated in both ISO-treated groups compared with vehicle, with intermittent delivery of ISO causing a greater increase in CTGF mRNA and protein than continuous infusion (Figures 5C and D).

\subsection{Effect of ISO on ROS production and NADPH oxidase}

LV sections stained with DHE revealed ISO-treated mice to have a marked increase in the amount of oxidation (Figure
6A). Western blot and qRT-PCR analysis both found NOX4 (an isoform of NADPH oxidase) expression to be significantly increased following ISO treatment in both delivery groups; however, this was to a greater extent in the injected ISO group (Figures 6B and C).

\section{Discussion}

A previous study demonstrated that the nature of the stress on the heart, rather than its duration, is the primary determinant of the resulting pathological phenotype [30]. The current study sought to address a similar hypothesis, inves- 

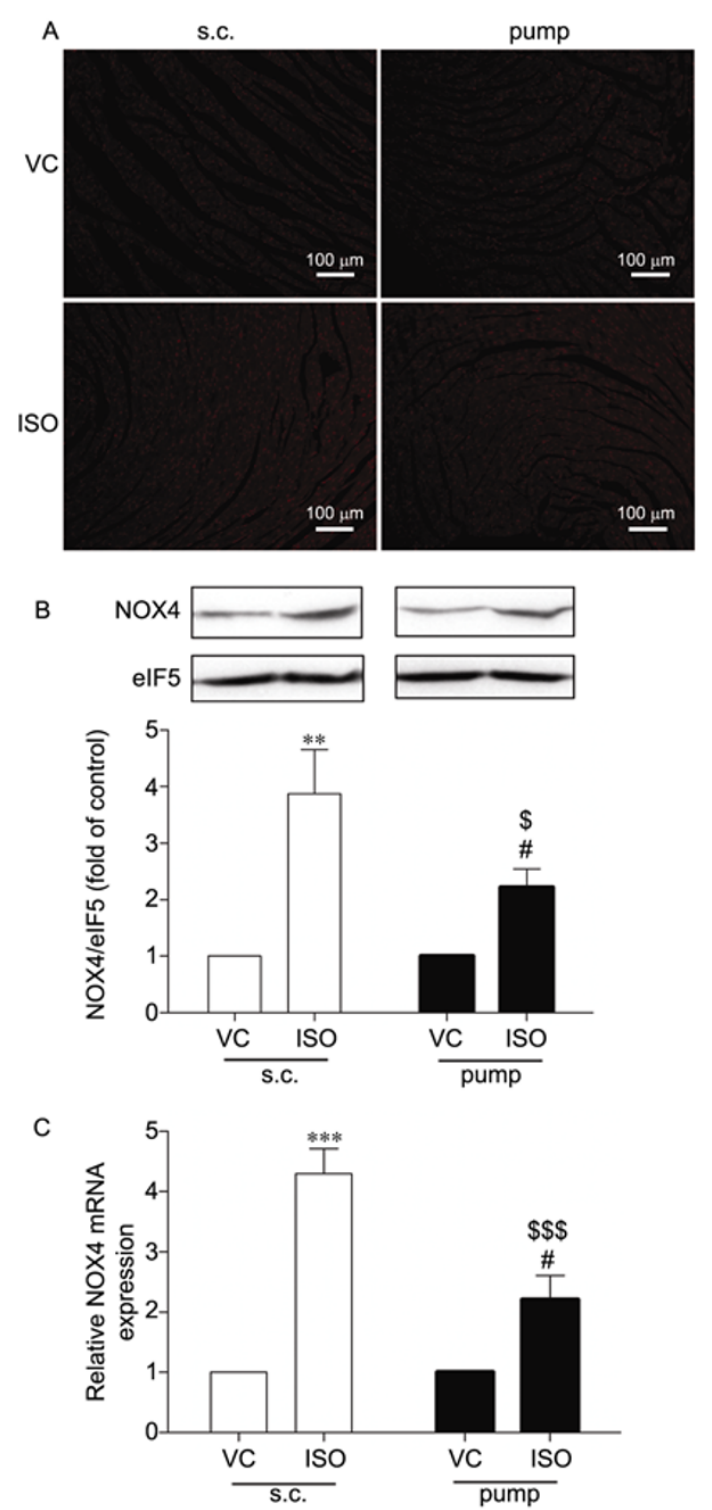

Figure 6 Effect of ISO on ROS production and NOX4 expression. A, Representative DHE staining in LV sections by fluorescent microscopy. B, NOX4 protein expression determined by Western blot $(n=4)$. Upper panels provide representative blots of analyzed and averaged results (lower graph). C, NOX4 mRNA expression determined by real-time PCR (n=6). s.c., subcutaneous injection; pump, continuous infusion via the osmotic pump. ** $P<0.01$, *** $P<0.001$ vs. VC s.c.; \# $P<0.05$ vs. VC pump; $\$ P<0.05$, $\$ \$ P<0.001$ ISO pump vs. ISO s.c.

tigating whether exposure to different patterns of the same stress could result in different pathological phenotypes in the heart. Here, we demonstrated that different patterns of $\beta$-AR stimulation resulted in similar levels of cardiac hypertrophy; however, significantly differed in their degree of cardiac fibrosis and cardiac dysfunction. Intermittent application of ISO was found to cause the greatest detrimental effects on the heart, including greater ROS production and expression of NOX4 compared with mice continuously infused. Taken together, these results indicate that intermittent
$\beta$-AR activation is more prone to induce maladaptive cardiac remodeling and dysfunction than sustained $\beta$-AR stimulation.

ISO, the potent, nonselective $\beta$-AR agonist used in the current study is well reported to have positive inotropic actions and dose-dependently increase heart rate [31, 32]. In our study, ISO-induced changes in heart rate were found to be dependent upon the two methods of drug delivery. Animals receiving ISO by subcutaneous injection produced the greatest increase in heart rate $(45 \%)$ at $1 \mathrm{~min}$, before reducing to become equal with basal levels after $30 \mathrm{~min}$. In contrast, the heart rate of animals receiving a continuous infusion of ISO via a minipump was elevated by $26 \%$ within the first minute of infusion and remained significantly elevated throughout the experimental period. The different responses in heart rate dependent upon the method of ISO delivery reflect that daily injections and continuous infusion of ISO provided a good model of intermittent and sustained $\beta$-AR stimulation, respectively. It is also apparent that animals receiving a bolus injection of ISO induced greater $\beta$-AR stimulation compared with continuous ISO infusion, despite receiving the same dose per day.

Studies observing the effects of ISO-induced cardiac remodeling to date have applied a wide range of doses and used various methods of administration. For example, Kitagawa Y et al. reported ISO to induce cardiac hypertrophy and fibrosis following continuous infusion of ISO at both $1.2 \mathrm{mg}$ $(\mathrm{kg} \mathrm{d})^{-1}$ and $\left.2.4 \mathrm{mg} \mathrm{(kg} \mathrm{d}\right)^{-1}$ by an osmotic pump [16]. Similarly, studies administering ISO by subcutaneous injection reported ISO to induce cardiac hypertrophy and fibrosis in a dose-dependent manner [17]. Consistent with these reports, the current study directly contrasted the two methods of ISO delivery, revealing the degree of cardiac hypertrophy to be equivalent following both patterns of $\beta$-AR stimulation. Similarly, Hohimer A R et al. reported that one subcutaneous injection of $20 \mu \mathrm{g} \mathrm{g}^{-1}$ ISO per day for 5 consecutive days caused increases in ventricular weight/body weight equivalent to 5 daily infusions at $20 \mu \mathrm{g}(\mathrm{g} \mathrm{d})^{-1}$ [33].

Increased collagen synthesis is considered to be an indicator of interstitial fibrosis. In the present study, we estimated collagen synthesis by both Sirius red staining and measuring the mRNA content of myocardial collagen-I and collagen-III. These analyses revealed that intermittent $\beta$-AR stimulation led to more severe interstitial fibrosis compared with sustained $\beta$-AR stimulation. Cardiac hemodynamic function is heavily influenced by cardiac fibrosis. Observing cardiac function in the current study, it was evident that 2 weeks of intermittent $\beta$-AR stimulation induced significant cardiac dysfunction. In contrast, continuous infusion of ISO increased cardiac systolic function, suggested to be due to the continuous positive inotropic effect of ISO. This result is consistent with a prior report infusing ISO by the osmotic pump to mice for 7 days [34]. Following removal of the ISO pump, systolic function was preserved, suggesting compen- 
satory pathological cardiac remodeling occurred. Together, these findings suggest that intermittent $\beta$-AR activation is more prone to induce maladaptive cardiac remodeling and dysfunction than sustained $\beta$-AR stimulation.

To elucidate the potential signaling pathways underlying the distinct actions of intermittent and sustained $\beta$-AR stimulation on cardiac remodeling, we examined signaling pathways known to be associated with cardiac remodeling. In our hands, both intermittent and sustained application of ISO caused equivalent activation of mTOR/p70S6k and MAPKs (including ERK, p38 and JNK). Recently, Xu Q et al. demonstrated that $\beta_{2}$-adrenoceptor stimulation provoked NADPH oxidase-derived ROS production in the heart, which contributed to cardiac inflammation, remodeling and heart failure [35]. Furthermore, there is increasing evidence that ROS-related signaling pathways can modulate CTGF synthesis, a key mediator of AngII-induced cardiac fibrosis [27]. The current study found that ROS production, as well as NOX4 and CTGF expression all increased with ISO treatment. Interestingly, intermittent ISO delivery caused greater ROS production, as well as increased NOX4 and CTGF expression compared with continuous ISO infusion. These results strongly suggest that a ROS-related pathway contributes to developing cardiac fibrosis and cardiac dysfunction, with intermittent application of ISO exacerbating the pathological effects. The intriguing disparate results following intermittent and sustained $\beta$-AR stimulation on ROS-related signaling proteins require further investigation, including determining the level of MDA and NADPH oxidase activity.

In conclusion, the current study demonstrates that both intermittent and sustained stimulation of $\beta$-AR cause cardiac hypertrophy to a similar extent. However, intermittent $\beta$-AR stimulation leads to more severe cardiac fibrosis and cardiac dysfunction compared with sustained $\beta$-AR stimulation. Our findings significantly further our understanding of $\beta$-AR stimulation in relation to cardiac pathophysiology, highlighting the importance of the mode of drug delivery at both the clinical and experimental setting.

The authors thank Dr. Du XiaoJun (Baker IDI Heart Diabetes Institute and Alfred Medical School, Monash University, Melbourne, Victoria, Australia) for his guidance and input. This work was supported by the National Basic Research Program of China (Grant No. 2011CB503903), the National Natural Science Foundation of China (Grant Nos. 81030001 and 30971161) and the International Cooperation and Exchange of the National Natural Science Foundation of China (Grant No. 30910103902).

1 Floras J S. Sympathetic activation in human heart failure: diverse mechanisms, therapeutic opportunities. Acta Physiol Scand, 2003, 177: 391-398

2 Lohse M J, Engelhardt S, Eschenhagen T. What is the role of beta-adrenergic signaling in heart failure. Circ Res, 2003, 93: 896-906

3 Singh K, Communal C, Sawyer D B, et al. Adrenergic regulation of myocardial apoptosis. Cardiovasc Res, 2000, 45: 713-719

4 Fowler M B, Laser J A, Hopkins G L, et al. Assessment of the beta-adrenergic receptor pathway in the intact failing human heart: progressive receptor down-regulation and subsensitivity to agonist response. Circulation, 1986, 74: 1290-1302

5 Goldspink D F, Burniston J G, Ellison G M, et al. Catecholamine-induced apoptosis and necrosis in cardiac and skeletal myocytes of the rat in vivo: the same or separate death pathways. Exp Physiol, 2004, 89: 407-416

6 Communal C, Singh K, Pimentel D R, et al. Norepinephrine stimulates apoptosis in adult rat ventricular myocytes by activation of the beta-adrenergic pathway. Circulation, 1998, 98: 1329-1334

7 Bos R, Mougenot N, Findji L, et al. Inhibition of catecholamine-induced cardiac fibrosis by an aldosterone antagonist. J Cardiovasc Pharmacol, 2005, 45: 8-13

8 Grassi G, Quarti-Trevano F, Dell'oro R, et al. Essential hypertension and the sympathetic nervous system. Neurol Sci, 2008, 29: S33-36

9 Flaa A, Eide I K, Kjeldsen S E, et al. Sympathoadrenal stress reactivity is a predictor of future blood pressure: an 18-year follow-up study. Hypertension, 2008, 52: 336-341

10 Sharkey S W, Lesser J R, Zenovich A G, et al. Acute and reversible cardiomyopathy provoked by stress in women from the United States. Circulation, 2005, 111: 472-479

11 Ueyama T, Kasamatsu K, Hano T, et al. Emotional stress induces transient left ventricular hypocontraction in the rat via activation of cardiac adrenoceptors: a possible animal model of 'tako-tsubo' cardiomyopathy. Circ J, 2002, 66: 712-713

12 Ueyama T, Senba E, Kasamatsu K, et al. Molecular mechanism of emotional stress-induced and catecholamine-induced heart attack. J Cardiovasc Pharmacol, 2003, 41: S115-118

13 Rona G, Chappel C I, Balazs T, et al. An infarct-like myocardial lesion and other toxic manifestations produced by isoproterenol in the rat. AMA Arch Pathol, 1959, 67: 443-455

14 Zbinden G, Moe R A. Pharmacological studies on heart muscle lesions induced by isoproterenol. Ann N Y Acad Sci, 1969, 156: 294-308

15 Kung H F, Blau M. Subcutaneous isoproterenol: a convenient rat model for early detection of myocardial necrosis. J Nucl Med, 1978, 19: 948-951

16 Kitagawa Y, Yamashita D, Ito H, et al. Reversible effects of isoproterenol-induced hypertrophy on in situ left ventricular function in rat hearts. Am J Physiol Heart Circ Physiol, 2004, 287: H277-285

17 Benjamin I J, Jalil J E, Tan L B, et al. Isoproterenol-induced myocardial fibrosis in relation to myocyte necrosis. Circ Res, 1989, 65: 657-670

18 Clerk A, Michael A, Sugden P H. Stimulation of the p38 mitogen-activated protein kinase pathway in neonatal rat ventricular myocytes by the $\mathrm{G}$ protein-coupled receptor agonists, endothelin-1 and phenylephrine: a role in cardiac myocyte hypertrophy. J Cell Biol, 1998, 142: 523-535

19 Bueno O F, De Windt L J, Tymitz K M, et al. The MEK1-ERK1/2 signaling pathway promotes compensated cardiac hypertrophy in transgenic mice. EMBO J, 2000, 19: 6341-6350

20 Ramirez M T, Sah V P, Zhao X L, et al. The MEKK-JNK pathway is stimulated by alphal-adrenergic receptor and ras activation and is associated with in vitro and in vivo cardiac hypertrophy. J Biol Chem, 1997, 272: 14057-14061

21 Boluyt M O, Zheng J S, Younes A, et al. Rapamycin inhibits alpha 1-adrenergic receptor-stimulated cardiac myocyte hypertrophy but not activation of hypertrophy-associated genes. Evidence for involvement of p70 S6 kinase. Circ Res, 1997, 81: 176-186

22 Sadoshima J, Izumo S. Rapamycin selectively inhibits angiotensin II-induced increase in protein synthesis in cardiac myocytes in vitro. Potential role of 70-kD S6 kinase in angiotensin II-induced cardiac hypertrophy. Circ Res, 1995, 77: 1040-1052

$23 \mathrm{Hu} \mathrm{Y,} \mathrm{Zhang} \mathrm{Y,} \mathrm{Venkitaramani} \mathrm{D} \mathrm{V,} \mathrm{et} \mathrm{al.} \mathrm{Translation} \mathrm{of} \mathrm{stri-}$ atal-enriched protein tyrosine phosphatase (STEP) after beta1-adrenergic receptor stimulation. J Neurochem, 2007, 103: 531-541

24 Pesce L, Comellas A, Sznajder J I. Beta-adrenergic agonists regulate Na-K-ATPase via p70S6k. Am J Physiol Lung Cell Mol Physiol, 
2003, 285: L802-807

25 Dhalla N S, Temsah R M, Netticadan T. Role of oxidative stress in cardiovascular diseases. J Hypertens, 2000, 18: 655-673

26 Zhang G X, Kimura S, Nishiyama A, et al. Cardiac oxidative stress in acute and chronic isoproterenol-infused rats. Cardiovasc Res, 2005, 65: $230-238$

27 Ruperez M, Lorenzo O, Blanco-Colio L M, et al. Connective tissue growth factor is a mediator of angiotensin II-induced fibrosis. Circulation, 2003, 108: 1499-1505

28 Wang J, Xu N, Feng X, et al. Targeted disruption of Smad4 in cardiomyocytes results in cardiac hypertrophy and heart failure. Circ Res, 2005, 97: 821-828

29 Liao W, Wang S, Han C, et al. 14-3-3 proteins regulate glycogen synthase 3beta phosphorylation and inhibit cardiomyocyte hypertrophy. FEBS J, 2005, 272: 1845-1854

30 Perrino C, Naga P S V, Mao L, et al. Intermittent pressure overload triggers hypertrophy-independent cardiac dysfunction and vascular rarefaction. J Clin Invest, 2006, 116: 1547-1560

31 Han Y S, Tveita T, Kondratiev T V, et al. Changes in cardiovascular beta-adrenoceptor responses during hypothermia. Cryobiology, 2008, 57: 246-250

32 Kralova E, Mokran T, Murin J, et al. Electrocardiography in two models of isoproterenol-induced left ventricular remodeling. Physiol Res, 2008, 57: S83-89

33 Hohimer A R, Davis L E, Hatton D C. Repeated daily injections and osmotic pump infusion of isoproterenol cause similar increases in cardiac mass but have different effects on blood pressure. Can J Physiol Pharmacol, 2005, 83: 191-197

34 Freund C, Schmidt-Ullrich R, Baurand A, et al. Requirement of nuclear factor-kappaB in angiotensin II- and isoproterenol-induced cardiac hypertrophy in vivo. Circulation, 2005, 111: 2319-2325

$35 \mathrm{Xu}$ Q, Dalic A, Fang L, et al. Myocardial oxidative stress contributes to transgenic beta-adrenoceptor activation-induced cardiomyopathy and heart failure. Br J Pharmacol, 2011, 162: 1012-1028

Open Access This article is distributed under the terms of the Creative Commons Attribution License which permits any use, distribution, and reproduction in any medium, provided the original author(s) and source are credited. 